\title{
Reparación endoscópica de encefalocele esfenoidal, secundario a persistencia del canal de Sternberg
}

\section{Endoscopic repair of sphenoid encephalocele, secondary to persistence of Sternberg's canal}

Roberto Arias A', Mariana Bogado².

\begin{abstract}
RESUMEN
Las fístulas de líquido cefalorraquídeo (FLCR) nasales se producen por la comunicación entre el espacio subaracnoídeo y el tracto aerodigestivo. Pueden ser adquiridas (secundarias a trauma o iatrogenia) o espontáneas. Éstas últimas pueden ser de origen congénito, tumoral o idiopáticas.

El canal lateral craneofaríngeo o de Sternberg se produce por la falta de fusión de los puntos de osificación del seno esfenoidal durante el período embrionario. Esta región, ubicada posterolateral a la pared inferior del seno esfenoidal, queda cubierta solamente por tejido conectivo, siendo la zona de menor resistencia de la base de cráneo.

La persistencia de este canal puede causar FLCR, especialmente cuando se asocia a elevación de la presión intracraneana y extensa neumatización. La cirugía endoscópica nasal es la técnica de elección para la resolución de estos casos.

En el presente artículo se realiza una revisión de la técnica quirúrgica endoscópica nasal para la resolución quirúrgica de encefaloceles secundarios a persistencia del canal de Sternberg, en base a dos casos clínicos que fueron sometidos a cirugía endoscópica nasal.
\end{abstract}

Palabras clave: Canal de Sternberg, encefalocele, fístula de líquido cefalorraquídeo.

\begin{abstract}
Nasal cerebrospinal fluid leaks are caused by a nasal communication between the subarachnoid space and the aerodigestive tract. They may be acquired (secondary to trauma or iatrogenic) or spontaneous. The latter can be congenital, tumoral or idiopathic. The lateral craniopharyngeal canal or Sternberg canal is caused by the lack of fusion of the points of ossification of the sphenoid sinus during the embryonic period. This region, located posterolateral to the bottom wall of the sphenoid sinus, is covered only by connective tissue, being the area of least resistance of the skull base. The persistence of
\end{abstract}

${ }^{1}$ Médico Otorrinolaringólogo. Servicio de Otorrinolaringología, Hospital del Salvador.

${ }^{2}$ Médico Residente de Otorrinolaringología, Hospital del Salvador.

Recibido el 20 de octubre, 2013. Aceptado el 21 de agosto, 2014. 
this canal can cause cerebrospinal fluid leaks, especially when associated with elevated intracranial pressure and extensive pneumatization. Nasal endoscopic surgery is the technique of choice for solving these cases.

This article is a review of the endoscopic surgical technique of encephaloceles secondary to persistence of Sternberg canal, based on two clinical cases.

Key words: Sternberg canal, Encephalocele, Cerebroespinal fluid leak.

\section{INTRODUCCIÓN}

Las fístulas de líquido cefalorraquídeo (FLCR) nasales se producen por la comunicación entre el espacio subaracnoideo y el tracto aerodigestivo $0^{1,2}$. Se pueden producir secundarias a cualquier proceso patológico que comprometa la pared lateral o superior del seno esfenoidal, pared superior del etmoides o lámina cribiforme, debido a que en esta región la duramadre está firmemente adherida a estas estructuras ${ }^{1-4}$.

Las FLCR pueden ser clasificadas según su etiología en adquiridas (secundarias a trauma 0 iatrogenia) o espontáneas. Estas últimas pueden ser de origen congénito, tumoral o idiopáticas (asociadas a hipertensión endocraneana) ${ }^{1-5}$. Otra forma de clasificarlas es en relación a la ubicación anatómica de las lesiones de la base de cráneo que las producen: tipo I-seno frontal, tipo Ila-lámina cribosa, tipo IIb-fóvea etmoidal, tipo III-seno esfenoidal ${ }^{1-6}$.

El canal lateral craneofaríngeo 0 de Sternberg se produce por la falta de fusión de los puntos de osificación del seno esfenoidal (entre alas mayores y preesfenoides 0 esfenoides anterior) durante el período embrionario $0^{7,8}$. Esta región, ubicada posterolateral a la pared inferior del seno esfenoidal, queda cubierta solamente por tejido conectivo, siendo la zona de menor resistencia de la base de cráneo. Este canal fue descrito por Sternberg en 1888 por primera vez $z^{8}$ y estaría presente hasta los 3-4 años de edad 9 . Se estima que $0,1 \%$ a $4 \%$ de los adultos presentan persistencia de este canal ${ }^{9}$. Se localiza generalmente lateral al agujero redondo mayor, por el cual atraviesa el nervio maxilar ${ }^{10}$.

La persistencia de este canal puede causar FLCR, especialmente cuando se asocia a elevación de la presión intracraneana y extensa neumatización $n^{1,2,7,9,10}$.

En el abordaje quirúrgico del seno esfenoidal se describen distintos accesos: transnasal directo, transnasal transetmoidoesfenoidal, etmoidoesfenoidal externo, transeptal, transantral y transcranial.

Los abordajes externos o intracraneales tienen la ventaja de permitir la visualización directa del defecto, mejor exposición de la zona operatoria y un adecuado manejo de las lesiones asociadas. Sin embargo, son cirugías de mayor duración y costo, se asocian a mayor frecuencia de complicaciones, mayor estadía hospitalaria y cicatrices ${ }^{9,10}$.

El abordaje transeptal tiene la ventaja que, al utilizar la línea media, permite una gran exposición de la zona media y paramediana, sin dejar cicatrices externas, permite corregir deformidades septales y puede complementarse con uso de microscopio.

El abordaje transnasal con apoyo endoscópico es considerado el gold standard en las lesiones ubicadas en la pared lateral del seno esfenoidal, con tasas de curación cercanas al 90\%. Es una cirugía de menor costo, con menos complicaciones y sin secuelas estéticas. Sin embargo, requiere de cirujanos con experiencia y es limitada en defectos múltiples ${ }^{9}$.

\section{EXPERIENCIA CLÍNICA}

\section{Caso 1}

Paciente mujer de 39 años, consulta en agosto de 2003 por cuadro de un mes de evolución de rinorrea acuosa por fosa nasal izquierda asociado a cefalea, sin otros síntomas. Es derivada a oftalmología desde atención primaria por posible obstrucción del conducto lacrimonasal, donde se objetiva rinorraquia que aumenta con valsalva. Es derivada al Instituto de Neurocirugía por posible FLCR. En la tomografía computarizada (TC) de cerebro se observa meningioma frontal derecho. Es sometida a craneotomía frontoparietal derecha con extirpación 
completa del meningioma, previa embolización de la arteria meníngea media derecha. Por persistencia posoperatoria de rinorraquia (confirmada por 2-transferrina), en octubre de 2003 es sometida a reparación subdural y extradural de brecha osteomeníngea a etmoides posterior, mediante craneotomía frontal y colocación de válvula derivativa ventrículo peritoneal. En agosto de 2006 presenta nuevamente rinorraquia por fosa nasal izquierda. En cisterno TC se observa FLCR, por lo que es sometida a cierre de brecha osteomeníngea mediante abordaje transpterigoideo. El año 2009 es hospitalizada por meningitis bacteriana aguda, evolucionando con amaurosis bilateral (atrofia óptica bilateral mayor a izquierda), hipoacusia sensorioneural y paraparesia de extremidades inferiores. En la resonancia magnética (RM) de encéfalo se observan cambios compatibles con meningoencefalitis parietal focal derecha, cambios posoperatorios frontales izquierdos y parietal derecho y defecto en la pared lateral izquierda del seno esfenoidal (canal de Sternberg) con pequeño encefalocele temporal medial secundario. El año 2011, presenta nuevamente meningitis bacteriana aguda y se constata FLCR activa al examen físico. No es posible identificar zona de brecha en cisterno TC, se realiza RM de cavidades paranasales y cerebro que evidencia meningocele en zona del canal de Sternberg. Es sometida a reparación de fístula por vía transnasal mediante técnica endoscópica (Figuras 2,3 y 4). No ha presentado nuevos episodios de rinorraquia.

\section{Caso 2}

Paciente mujer de 62 años, consulta por cuadro de 3 meses de evolución con rinorrea unilateral intermitente, asociado a cefalea. En la TC de cavidades paranasales y RM se observa encefalocele en zona del canal de Sternberg a derecha.

Es sometida a cierre de la FLCR con equipo compuesto por otorrinolaringólogo y neurocirujano, por vía transnasal mediante técnica endoscópica. No ha presentado nuevos episodios de rinorraquia.

En ambos casos la técnica quirúrgica utilizada es la que se describe a continuación:

Para la cirugía endonasal con apoyo endoscópico de base de cráneo, el acceso debe ser realizado idealmente con dos cirujanos, un neurocirujano y un otorrinolaringólogo. Se utiliza un endoscopio de $30^{\circ}$.

Para el abordaje quirúrgico se requiere.

1. Acceso nasal endoscópico con exposición amplia del seno esfenoidal.

2. Identificación de zona de encefalocele.

3. Reducción de encefalocele con bipolar, exposición de zona de brecha en toda su periferia.

4. Confección de tapón de cartílago y grasa (Figura 1).

5. Plastía con duramadre liofilizada, Duragen ${ }^{\circledR}$ y Beriplast $^{\circledR}$.

6. Sellado del seno esfenoidal con material biológico.

7. Reposición del tabique en posición anatómica.

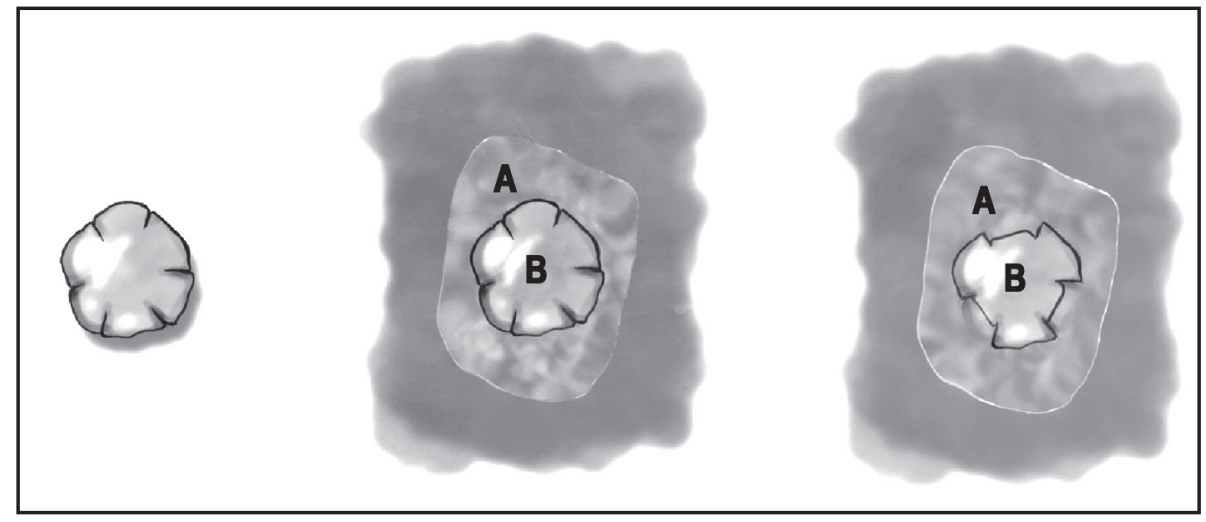

Figura 1. Esquema de cierre del defecto óseo. En A: tejido adiposo. En B: cartílago. 

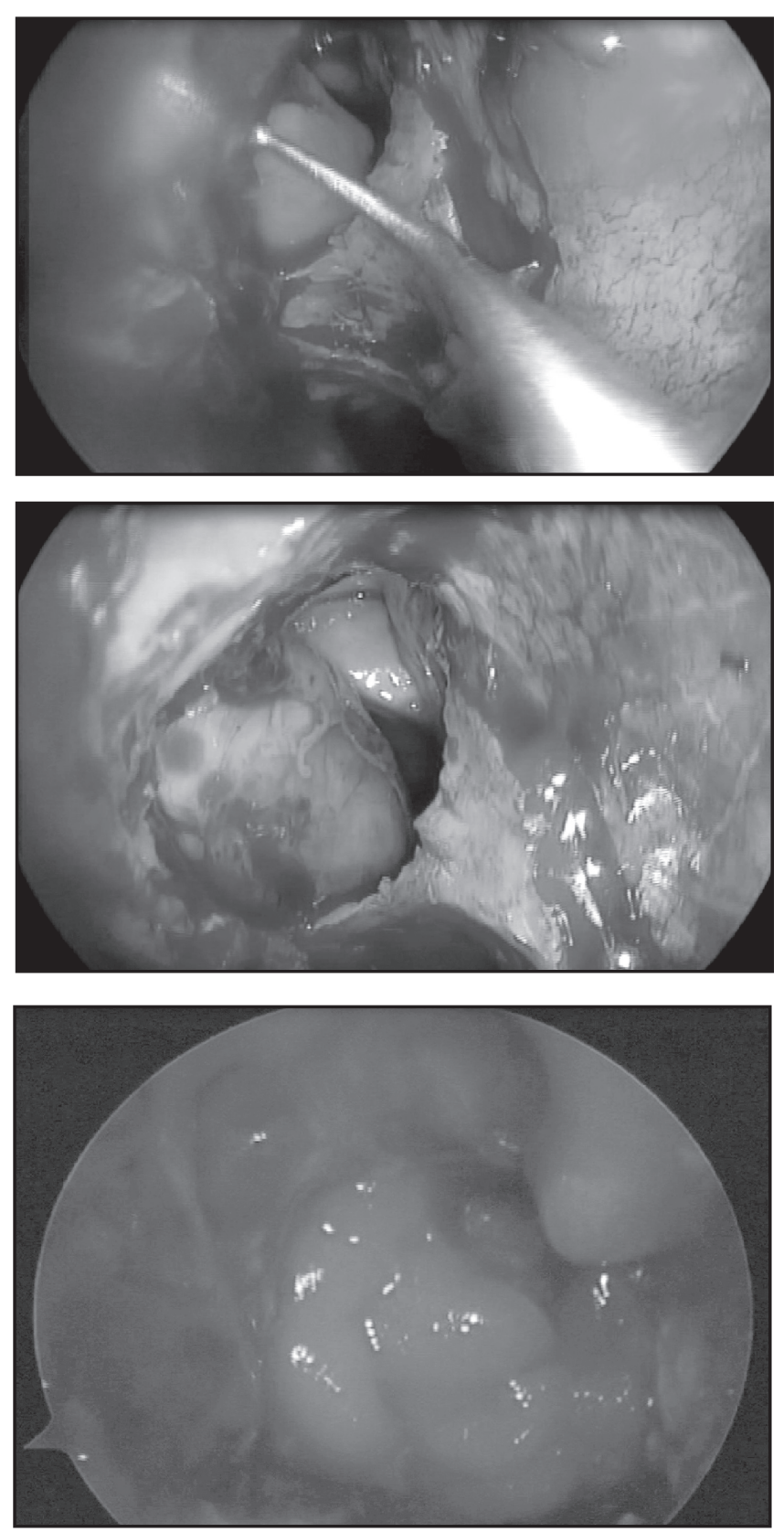

Figuras 2, 3, 4. Cierre bajo visión endoscópica de encefalocele esfenoidal.

Ambos pacientes fueron manejados en el posoperatorio con medidas para disminuir la presión y producción de líquido cefalorráquideo: reposo con cabeza en $30^{\circ}$, evitar esfuerzos y valsalva y se indicó acetazolamida $(250 \mathrm{mg} / 8$ hrs por vía oral), los pacientes fueron hospitalizados por una semana y no se observó complicaciones posoperatorias. No fue necesaria la realización de drenaje lumbar dada la adecuada evolución. 


\section{DISCUSIÓN}

Los encefaloceles y las FLCR que comprometen el receso lateral del esfenoides son relativamente poco frecuentes. Los encefaloceles de gran tamaño generalmente se diagnostican en el período prenatal 0 al nacimiento como consecuencia de un gran defecto del tubo neural, sin embargo, encefaloceles de menor tamaño pueden pasar desapercibidos por años o producirse en forma espontánea como resultado de un aumento de la presión intracraneana que vence la zona débil del canal de Sternberg ${ }^{10-12}$.

Clínicamente los pacientes con encefaloceles del receso lateral del esfenoides pueden presentar cefalea, desequilibrio, mareos, rinorraquia unilateral, diplopía o meningitis, o ser completamente asintomáticos, por lo que pueden pasar desapercibidos por años o ser un hallazgo imagenológico incidental ${ }^{10,11}$.

Independiente de la clínica presentada, los pacientes con encefalocele de la pared lateral del esfenoides deben ser sometidos a reparación quirúrgica, con la finalidad de aliviar la sintomatología y evitar complicaciones como meningitis.

La reparación quirúrgica de las lesiones que comprometen el receso lateral del esfenoides representan un desafío mayor que las otras fístulas nasales, debido a la dificultad para visualizarla y acceder a esta zona por vía transnasal, especialmente si existe gran neumatización del seno esfenoidal. Las técnicas de abordaje externas requieren una cirugía de mayor extensión, se asocian a mayor morbilidad y generalmente requieren más de una cirugía para lograr intentar su cierre ${ }^{10,11}$.

Algunos autores plantean el uso del colgajo de Hadad-Bassagaisteguy para cubrir el defecto después de la cirugía, el cual debe ser tomado al comienzo de la cirugía, desde el lado contralateral al acceso transpterigoideo ${ }^{13}$. Este colgajo es neurovascular pediculado e incluye mucoperiostio del tabique nasal y mucopericondrio de la arteria nasoseptal. El uso de este colgajo ha demostrado ser muy versátil para cubrir los defectos de la fosa anterior, media, clival y paraselar, disminuyendo la incidencia de FLCR posoperatoria y su uso es sugerido para la reconstrucción de grandes defectos durales $^{14-16}$, por lo que su uso en encefaloceles ubicados en el receso lateral sería de gran utilidad.
Los casos expuestos demuestran la complejidad del acceso a esta zona, y los buenos resultados obtenidos con el cierre de la FLCR por acceso transnasal mediante técnica endoscópica.

\section{BIBLIOGRAFÍA}

1. Castell Luis F. Manejo de fístulas de LCR de la lámela lateral de la lámina cribosa. Rev Otorrinolaringol Cir Cabeza Cuello 2007; 67(1): 46-52.

2. Abarca A Alfredo, Pardo J Javiera, Lemp M Melchor, Stott C Carlos. Reparación endoscópica endonasal de fístulas de líquido cefalorraquídeo no quirúrgicas: Experiencia Hospital Clínico Universidad de Chile. Rev Otorrinolaringol Cir Cabeza Cuello 2010; 70(3): 205-14.

3. Kerr J, Chu F, Bayles S. Cerebrospinal fluid rhinorrhea: diagnosis and management. Otolaryngol Clin North Am 2005; 38(4): 597611.

4. Martin T, LoehrL T. Endoscopic CSF leak repair. Current Opinion in Otolaryngology \& Head and Neck Surgery 2007; 15: 35-9.

5. McMains K, Gross C, Kountakis S. Endoscopic management of cerebrospinal fluid rhinorrhea. Laryngoscope 2004; 114: 1833-7.

6. Meco C, Oberascher G. Comprehensive algorithm for skull base dural lesion and cerebrospinal fluid fistula diagnosis. Laryngoscope 2004; 114 : 991-9.

7. Al-Nashar I, Carrau R, Herrera A, Snyderman C. Endoscopic transnasal transpterygopalatine fossa approach to the lateral recess of the sphenoid sinus. Laryngoscope 2004; 114(3): 528-32.

8. Sternberg M. Ein bisher beschriebener Kanal im Keilbein des Menschen. Anat Anz 1888; 23 : 784-6.

9. Schick B, Brors D, Prescher A. Sternberg's canal - cause of congenital sphenoidal meningocele. Eur Arch Otorhinolaringol 2000; 257(8): 430-2.

10. Schmidt R, Choudhry 0, Raviv J y cols. Surgical nuances for the endoscopic endonasal transpteryoid approach to lateral sphenoid sinus encephaloceles. Neurosurg Focus 2012; 32(6): E5: 1-8.

11. Rossi M, Martín C, Labella T. Asociación entre fístula de líquido cefalorraquídeo y persistencia 
del canal de Sternberg: ¿coincidencia o causa? Acta Otorrinolaringológica Española 2012; 63 (2): 144-6.

12. BoLgeR W. Endoscopic Transpterygoid approach to the lateral sphenoid recess: surgical approach and clinical experience. Otolaryngol Head Neck Surg 2005; 133: 20-6.

13. Kasemsiri P, Carrau R, Prevedello D y cols. "Así lo hago yo": accesos endoscópicos endonasales transpterigoideos. Acta Otorrinolaringológica y Cirugía de Cebeza y Cuello 2012; 40 (1): 119-24.

14. Hadad Gustavo, Luis Bassagasteguy, Ricardo L Carrau y cols. A Novel Reconstructive
Technique After Endoscopic Expanded Endonasal Approaches: Vascular Pedicle Nasoseptal Flap. The Laryngoscope 2006; 116 (10): 1882-6.

15. Hadad Gustavo, Carlos M Rivera-Serrano, Luis H Bassagaisteguy, y cols. Anterior Pedicle Lateral Nasal Wall Flap: a Novel Technique for the Reconstruction of Anterior Skull Base Defects. The Laryngoscope 2011; 121 (8): 1606-10.

16. Herrera A, Ospina J, Téllez P, Feo L. Abordaje endoscópico transnasal para la resección de tumores de la base de cráneo: nuestra experiencia. Acta Otorrinolaringológica y Cirugía de Cabeza y Cuello 2012; 40(1): 125-32.

Dirección: Roberto Arias A. Hospital del Salvador

Avda. El Salvador 364. Providencia, Santiago, Chile

E mail: ropat010@hotmail.com 